\title{
Avaliação do Ensino nos Cursos de Pós-Graduação em Engenharia: Um Enfoque Quantitativo de Avaliação em Conjunto
}

\author{
Lidia Angulo Meza \\ Instituto de Ciência e Tecnologia - Universidade Veiga de Almeida \\ Rua Ibituruna, 108, $4^{\circ}$ andar, Maracanã, 20271-020, Rio de Janeiro, RJ \\ lidia@lab.uva.br
}

\section{Eliane Gonçalves Gomes}

Programa de Engenharia de Produção - COPPE - Universidade Federal do Rio de Janeiro Embrapa Monitoramento por Satélite

Av. Dr. Júlio Soares de Arruda, 803, Parque São Quirino, 13088-300, Campinas, SP eliane@cnpm.embrapa.br

\section{Luiz Biondi Neto}

Departamento de Engenharia Eletrônica e Telecomunicações - Universidade do Estado do Rio de Janeiro

Rua São Francisco Xavier, 524, Bl. E, Sala 5025, Maracanã, 20550-900, Rio de Janeiro, RJ lbiondi@uerj.br

\section{Pedro Henrique Gouvêia Coelho}

Departamento de Engenharia Eletrônica e Telecomunicações - Universidade do Estado do Rio de Janeiro

Rua São Francisco Xavier, 524, Bl. E, Sala 5025, Maracanã, 20550-900, Rio de Janeiro, RJ phcoelho@uerj.br

\begin{abstract}
Resumo
Os cursos nacionais de pós-graduação são avaliados periodicamente por diferentes entidades, segundo critérios nem sempre claros. Esses critérios tentam considerar uma grande quantidade de variáveis, o que acarreta uma alta subjetividade nas avaliações de produtividade. Para quantificar e agregar estas variáveis em único índice há a necessidade de impor pesos, cuja subjetividade pode ser causa de desconforto e não aceitação dos resultados. Uma das formas de avaliar considerando-se várias variáveis é utilizar a Análise Envoltória de Dados (Data Envelopment Analysis - DEA), uma abordagem quantitativa e comparada, sem imposição de pesos. Por outro lado, para evitar alguns problemas decorrentes da auto-avaliação característica dessa análise, pode-se empregar uma técnica adicional, chamada de Avaliação Cruzada, que, em palavras simples, não é mais do que a avaliação feita pelo conjunto de unidades em avaliação. Este artigo pretende mostrar uma forma de medir a eficiência de unidades de ensino, especificamente, dos Programas de Pós-Graduação em Engenharia da Universidade Federal do Rio de Janeiro, em relação à produção de seus recursos humanos. Para tal, é utilizada a abordagem por DEA, cujos resultados são refinados pela Avaliação Cruzada, de modo a permitir uma avaliação em conjunto.
\end{abstract}

Palavras-chave: Pós-graduação - Análise Envoltória de Dados - Avaliação em conjunto. 


\section{Introdução}

nível Os cursos de pós-graduação a periodicamente por diferentes entidades, segundo critérios que nem sempre são claros. Esses critérios buscam medir, dentre outros, a produtividade acadêmica (incluindo-se qualidade e quantidade da produção científica), a dedicação do corpo docente, o tempo para conclusão do curso etc. A quantificação da excelência acadêmica não é geralmente realizada; é feita de forma qualitativa. Desta forma, as avaliações de produtividade em educação são normalmente subjetivas, em especial devido à grande quantidade de variáveis a serem consideradas. Para quantificar e agregar estas variáveis em único índice há a necessidade da imposição de pesos, cuja subjetividade pode ser causa de desconforto e não aceitação dos resultados.

Por outro lado, a avaliação educacional deve ser quantitativa e comparada (Boclin, 1999). A abordagem por Análise Envoltória de Dados (Data Envelopment Analysis - DEA) (Charnes et al., 1978) apresenta estas duas características em consonância, sem impor pesos, e alia a subjetividade da avaliação qualitativa com a objetividade da avaliação quantitativa de DEA (Soares de Mello et al., 2001b).

No entanto, os pesos atribuídos pelos modelos DEA clássicos são calculados através de um problema de programação linear, de forma que cada unidade em avaliação (Decision Making Unit - DMU) se beneficie com a melhor combinação de pesos, maximizando sua eficiência. Assim, para evitar que cada unidade em avaliação escolha seu melhor índice sem outras considerações, pode-se introduzir uma técnica adicional, chamada de Avaliação Cruzada, que, em palavras simples, pode ser vista como uma avaliação feita pelas outras unidades, isto é, uma avaliação de conjunto.

Por outro lado, a Análise de Envoltória de Dados tem sido utilizada para avaliar a eficiência de unidades educacionais. O trabalho de Soares de Mello et al. (2001a) apresenta DEA como uma ferramenta multicritério com aplicação em educação. Turmas de cálculo foram avaliadas utilizando DEA em Soares de Mello et al. (2002b). Gomes et al. (2002) utilizam a abordagem DEA para calcular a produtividade científica dos programas de pós-graduação em Engenharia utilizando uma técnica adicional, chamada restrições aos pesos, para refinar os resultados. Em Soares de Mello et al. (2002a) é feita uma análise DEA para analisar a qualidade e produtividade dos mesmos programas de pósgraduação.

No presente artigo apresenta-se uma forma de avaliação de eficiência dos Programas de Pós-Graduação em Engenharia da Universidade Federal do Rio de Janeiro, em relação à produção de seus recursos humanos (professores e funcionários), traduzida em teses defendidas, projetos, créditos ministrados e publicações. Esta avaliação é feita segundo duas óticas: como a unidade se avalia e como as outras unidades a avaliam.

\section{Análise Envoltória de Dados - DEA}

A Análise Envoltória de Dados (Data Envelopment Analysis - DEA), desenvolvida originalmente por Charnes et al. (1978) no contexto da avaliação de escolas, é um método que usa programação linear para determinar eficiências comparativas de Unidades de Tomada de Decisão (Decision Making Units - DMUs), em situações em que não seja preponderante ou não se deseja considerar somente o aspecto financeiro (Gomes et al., 2001). A eficiência relativa de uma DMU é definida como a razão da soma ponderada de seus produtos (outputs) pela soma ponderada dos insumos necessários para gerá-los (inputs).

Ao contrário dos modelos tradicionais de apoio à decisão com vários critérios, não há a figura de um 
decisor arbitrário que escolhe os pesos para cada fator de ponderação; eles são obtidos pelo próprio modelo matemático. Para isso, é resolvido um problema de programação fracionária que atribui a cada DMU os pesos que maximizam a sua eficiência. Assim, os pesos são diferentes e o mais benevolentes possíveis para cada unidade em estudo. Portanto, a abordagem por DEA impede que os responsáveis por uma unidade cuja avaliação não tenha sido boa argumentem que tal situação se deveu a uma escolha casuística de pesos.

Os modelos DEA estão baseados em problemas de programação linear para determinar os pesos de cada variável para determinar um índice de eficiência multidimensional ponderado. $\mathrm{O}$ modelo inicialmente desenvolvido, chamado de modelo DEA CCR (ㅡharnes et al., 1978), o qual considera retornos constantes de escala. A formulação desse modelo é apresentada em (1), previamente linearizada. Outros modelos que consideram diferentes escalas de operação, além de aplicações a situações reais podem ser encontrados em Lins e Angulo-Meza (2000) e em Cooper et al. (2000).

$$
\begin{aligned}
& \max h_{o}=\sum_{j=1}^{s} u_{j} y_{j o} \\
& \text { sujeito } a \\
& \sum_{i=1}^{m} v_{i} x_{i o}=1 \\
& \sum_{j=1}^{s} u_{j} y_{j k}-\sum_{i=1}^{m} v_{i} x_{i k} \leq 0, \quad k=1, \ldots, n \\
& u_{j}, v_{i} \geq 0 \quad \forall i, j
\end{aligned}
$$

Para o modelo (1), com $n$ DMUs, $m$ inputs e $s$ outputs, $h_{o}$ é a eficiência da DMU o em análise; $x_{i k}$ representa o input $i$ da DMU $k, y_{j k}$ representa o output $j$ da DMU $k ; v_{i}$ é o peso atribuído ao input $i, u_{j}$ é o peso atribuído ao output $j$. Esse modelo deve ser resolvido para cada DMU.

Neste artigo, em uma primeira etapa foi utilizado o modelo DEA CCR apresentado. A escolha desse modelo é justificada pelo fato de não se presumir a existência de efeitos de escala entre a defesa de teses, a publicação científica dos seus resultados, projetos e créditos ministrados.

A estrutura matemática dos modelos DEA faz com que, freqüentemente, uma DMU seja considerada eficiente por serem atribuídos pesos nulos a algumas variáveis. Isso significa que essas variáveis são desconsideradas na avaliação da eficiência daquela unidade, o que pode acarretar uma avaliação incompleta. No caso em estudo, a quantidade de variáveis a serem utilizadas em relação à quantidade de DMUs são indícios de que a situação anteriormente mencionada acontecerá, assim como uma pouca discriminação entre unidades eficientes, já que há uma recomendação empírica de que o número de DMUs seja o triplo ou o quádruplo do número de variáveis. González-Arraya (2003) aconselha que o número de variáveis seja cinco vezes maior que o número de DMUs. Logo, para obter resultados mais consistentes, é necessário usar técnicas adicionais (Angulo-Meza e Lins, 2002), tal como foi feito no trabalho de Soares de Mello et al. (2002a). Neste artigo, optou-se pelo uso da Avaliação Cruzada.

A Avaliação Cruzada é uma técnica que permite solucionar os dois problemas anteriormente mencionados, a saber, excesso de pesos zero arbitrados a algumas variáveis (desconsiderando-as da análise) e a baixa discriminação entre 
unidades eficientes (devido à relação quantidade de variáveis - número de DMUs). Por outro lado, essa técnica permite uma avaliação de conjunto: todas as unidades avaliam e são avaliadas pelas outras unidades, o que gera uma melhor aceitação dos resultados do modelo.

$$
E_{k s}=\frac{\sum_{i} u_{i k} y_{i s}}{\sum_{j} v_{j k} x_{j s}}
$$

Cabe destacar que para as DMUs eficientes os pesos apresentados não são únicos, já que os PPLs que determinam a eficiência de cada DMU apresentam múltiplas soluções ótimas nestes pontos. Para tais DMUs softwares de DEA diferentes podem encontrar conjuntos de pesos diferentes e que mantenham a DMU com eficiência 100\%. Para escolher entre os vários possíveis valores para os pesos ótimos de cada DMU,
Matematicamente, a avaliação cruzada utiliza os pesos atribuídos a cada DMU $k, v_{i k}$ e $u_{j k}$, segundo o modelo escolhido, e os utiliza para avaliar as outras unidades, conforme mostrado em (2), onde $E_{k s}$ é a eficiência da DMU $s$ avaliada segundo os pesos da DMU $k$.

arbitra-se que estes, quando aplicados às outras DMUs, devem minimizar a sua eficiência (formulação agressiva) ou, ao contrário, maximizá-la (formulação benevolente). Doyle e Green (1994) estabeleceram o PPL apresentado em (3) para o cálculo dos pesos na formulação agressiva, no qual a eficiência da DMU $s$ usando os pesos da DMU $k$ é dada em (2).

$$
\begin{aligned}
& \operatorname{Min} \sum_{i}\left(u_{i k} \sum_{s \neq k} y_{i s}\right)-\sum_{j}\left(v_{k j} \sum_{s \neq k} x_{j s}\right) \\
& \text { sujeito } a \\
& \sum_{j} v_{j k} x_{j k}=1 \\
& \sum_{i} u_{i k} y_{i k}-E_{k k} \sum_{j} v_{j k} x_{j k}=0 \\
& \sum_{i} u_{i k} y_{i s}-\sum_{j} v_{j k} x_{j s} \leq 0, \forall s \neq k \\
& u_{i k}, v_{j k} \geq 0
\end{aligned}
$$

Assim, o processo de avaliação é feito em duas etapas: primeiro, determina-se a eficiência segundo o modelo escolhido, neste caso o CCR, e, segundo, determinam-se os pesos segundo o modelo (3). Com as eficiências calculadas constrói-se uma matriz de eficiências cruzadas, na qual a média de cada coluna é a avaliação cruzada, $e_{s}$, ou seja, a avaliação do conjunto da unidade $s$ (tabela 1). 
Tabela 1. Matriz de Eficiências Cruzadas.

\begin{tabular}{|c|ccccccc|}
\cline { 2 - 8 } \multicolumn{1}{c|}{} & 1 & 2 & 3 & 4 & 5 & $\ldots$ & $\mathrm{n}$ \\
\hline 1 & $E_{11}$ & $E_{12}$ & $E_{13}$ & $E_{14}$ & $E_{15}$ & $\ldots$ & $E_{1 n}$ \\
2 & $E_{21}$ & $E_{22}$ & $E_{23}$ & $E_{24}$ & $E_{25}$ & $\ldots$ & $E_{2 n}$ \\
3 & $E_{31}$ & $E_{32}$ & $E_{33}$ & $E_{34}$ & $E_{35}$ & $\ldots$ & $E_{3 n}$ \\
4 & $E_{41}$ & $E_{42}$ & $E_{43}$ & $E_{44}$ & $E_{45}$ & $\ldots$ & $E_{4 n}$ \\
5 & $E_{51}$ & $E_{52}$ & $E_{53}$ & $E_{54}$ & $E_{55}$ & $\ldots$ & $E_{5 n}$ \\
$\ldots$ & $\ldots$ & $\ldots$ & $\ldots$ & $\ldots$ & $\ldots$ & $\ldots$ & $\ldots$ \\
$\mathrm{n}$ & $E_{n 1}$ & $E_{n 2}$ & $E_{n 3}$ & $E_{n 4}$ & $E_{n 5}$ & $\ldots$ & $E_{n n}$ \\
\hline & $e_{1}$ & $e_{2}$ & $e_{3}$ & $e_{4}$ & $e_{5}$ & $\ldots$ & $e_{n}$ \\
\hline
\end{tabular}

Maiores detalhes sobre Avaliação Cruzada podem ser encontrados em Angulo-Meza (1998) e Lins e AnguloMeza (2000).

\section{Avaliação de Unidades de Ensino: Programas de Pós- Graduação em Engenharia da Universidade Federal do Rio de Janeiro}

\subsection{Unidades de avaliação - DMUs}

Para esta análise foram selecionados os doze Programas de PósGraduação em Engenharia, sob coordenação do Instituto Alberto Luiz Coimbra de Pós-Graduação e Pesquisa de Engenharia (COPPE), com sede na Universidade Federal do Rio de Janeiro. São eles: Programa de Engenharia Biomédica (PEB), Programa de Engenharia Civil (PEC), Programa de Engenharia Elétrica (PEE), Programa de Engenharia Mecânica (PEM), Programa de Engenharia de Materiais e Metalúrgica (PEMM), Programa de Engenharia Nuclear (PEN), Programa de Engenharia Naval e Oceânica (PENO), Programa de Engenharia de Produção (PEP), Programa de Engenharia Química (PEQ), Programa de Engenharia de Sistemas e Computação (PESC), Programa de Engenharia de Transportes (PET) e Programa de Planejamento Energético (PPE). Esses programas representam as DMUs do modelo DEA.

\subsection{Variáveis da análise}

Dado que o objetivo da análise é a avaliação da eficiência dos recursos humanos, têm-se como recursos (inputs) a quantidade de professores e funcionários de cada programa e, como produtos (outputs) os créditos ministrados, os projetos (em milhares de reais), as teses defendidas (mestrado e doutorado) e as publicações. Neste caso é utilizada a pontuação dada pela CAD (Comissão de Avaliação de Docentes) para cada tipo de publicação dos professores.

Os dados utilizados referem-se ao somatório das variáveis para o período compreendido entre 1995 e 1997 (inclusive) apresentados na Tabela 2. O uso de dados atualizados não foi possível devido à falta de acesso aos mesmos. No entanto, este fato não descaracteriza a importância deste artigo, cujo objetivo principal é mostrar a utilidade de DEA, refinada com a técnica de Avaliação Cruzada, para avaliação de unidades de ensino, especialmente por ser uma abordagem que considera uma avaliação de conjunto. Assim, o modelo DEA CCR é constituído de 12 DMUs, 2 inputs e 4 outputs. 
Tabela 2. Dados para os programas.

\begin{tabular}{ccccccc}
\hline DMU & Projetos & Publicações & Teses & \multicolumn{2}{c}{ Créditos } & Professores Funcionários \\
\hline PEB & 130336,86 & 68,27 & 11,67 & 90,33 & 13,00 & 14,00 \\
PEC & 1790618,06 & 234,43 & 36,00 & 300,33 & 53,33 & 64,00 \\
PEE & 989332,43 & 120,70 & 21,67 & 152,67 & 26,00 & 14,33 \\
PEM & 338478,86 & 49,77 & 16,33 & 97,00 & 26,33 & 13,33 \\
PEMM & 1623378,54 & 117,43 & 20,67 & 141,33 & 33,00 & 34,33 \\
PEN & 540532,24 & 37,97 & 9,33 & 96,50 & 15,33 & 36,33 \\
PEO & 723597,18 & 24,47 & 13,67 & 149,00 & 24,33 & 17,33 \\
PEP & 982725,17 & 65,97 & 59,33 & 223,33 & 47,33 & 12,67 \\
PEQ & 1037699,70 & 222,67 & 27,67 & 121,00 & 29,67 & 36,67 \\
PESC & 1649750,15 & 116,63 & 36,33 & 167,00 & 28,33 & 24,00 \\
PET & 885904,46 & 60,23 & 14,33 & 95,67 & 11,33 & 5,33 \\
PPE & 1367673,21 & 36,57 & 11,67 & 50,83 & 12,00 & 10,33 \\
\hline
\end{tabular}

\subsection{Aplicação do enfoque DEA}

\subsubsection{Modelo DEA CCR}

Os resultados utilizando o modelo DEA CCR (eficiências e pesos das variáveis) e o software What's Best (www.lindo.com) são apresentados na Tabela 3.

Observa-se que o número de unidades $100 \%$ eficientes é grande (devido à já comentada relação entre o número de variáveis e o número de DMUs), o que dificulta a ordenação das unidades. Ressalta-se ainda o grande número de variáveis com peso zero (de três a cinco variáveis), o que significa que estas variáveis (critérios) são desconsideradas na determinação do índice de eficiência. Desta forma, tornase relevante um refinamento do modelo usado.

Tabela 3. Eficiência dos programas e pesos das variáveis.

\begin{tabular}{cccccccc}
\hline \multirow{2}{*}{ Programa } & Eficiência & \multicolumn{7}{c}{ Pesos das variáveis } \\
\cline { 3 - 7 } & CCR & Projetos & Publicações & Teses & Créditos & Professores & Funcionários \\
\hline PEB & 0,915 & 0,00000 & 0,00881 & 0,00000 & 0,00442 & 0,08410 & 0,00000 \\
PEC & 0,756 & 0,00000 & 0,00260 & 0,00000 & 0,00130 & 0,02480 & 0,00000 \\
PEE & 0,837 & 0,00000 & 0,00829 & 0,00000 & 0,00000 & 0,03290 & 0,02369 \\
PEM & 0,490 & 0,00000 & 0,00000 & 0,06124 & 0,00000 & 0,07611 & 0,00286 \\
PEMM & 0,652 & 0,00025 & 0,00501 & 0,00000 & 0,00000 & 0,04646 & 0,00000 \\
PEN & 0,745 & 0,00000 & 0,00000 & 0,00000 & 0,01036 & 0,08750 & 0,00000 \\
PENO & 0,725 & 0,00000 & 0,00000 & 0,00000 & 0,00671 & 0,05667 & 0,00000 \\
PEP & 1,000 & 0,00000 & 0,00000 & 0,00069 & 0,00429 & 0,00000 & 0,07893 \\
PEQ & 1,000 & 0,00018 & 0,00363 & 0,00000 & 0,00000 & 0,03370 & 0,00000 \\
PESC & 1,000 & 0,00000 & 0,00000 & 0,02753 & 0,00000 & 0,03421 & 0,00129 \\
PET & 1,000 & 0,00000 & 0,00000 & 0,00165 & 0,01021 & 0,00000 & 0,18762 \\
PPE & 1,000 & 0,00073 & 0,00000 & 0,00000 & 0,00000 & 0,02564 & 0,06701 \\
\hline
\end{tabular}

\subsubsection{Avaliação Cruzada}

Na literatura de DEA há algumas formas para contornar o problema do excesso de pesos nulos. Uma delas é introduzir restrições aos pesos no modelo (Angulo-Meza e Lins, 2002), o que implica em ter um consenso sobre a importância relativa de cada variável na análise. No estudo de caso deste artigo, isso não foi possível: cada programa considerava como importantes variáveis diferentes, especialmente quando a performance em uma determinada variável era muito favorável.

Esses pareceres conflitantes levaram à adoção da Avaliação Cruzada como estratégia alternativa, já que se, por um lado, não precisa de informação adicional por parte dos especialistas, o 
que evita a falta de consenso, por outro é uma forma de avaliar em conjunto, ou seja, considera os diferentes pareceres ao usar os pesos das variáveis encontrados pelos PPLs de DEA. Além dessas vantagens, a Avaliação Cruzada permite incrementar a discriminação entre os programas de pós-graduação eficientes.

Na Tabela 4, apresenta-se a matriz de eficiências cruzadas, calculadas utilizando-se a formulação agressiva apresentada em (3). Nessa tabela, observa-se uma melhor ordenação das
DMUs e uma avaliação mais completa por desconsiderar um menor número de variáveis. Na última linha, $e_{k}$ é a média da coluna, por exemplo, $e_{\mathrm{PPE}}$ é a média de todas as avaliações do programa de pósgraduação PPE feita por todos os programas, incluindo a própria avaliação de PPE. Note-se, ainda, que a diagonal da matriz representa a eficiência obtida com o modelo DEA CCR (resultados da Tabela 3).

Tabela 4. Matriz de Eficiências Cruzadas.

\begin{tabular}{|c|cccccccccccc|}
\cline { 2 - 10 } \multicolumn{1}{c|}{} & PEB & PEC & PEE & PEM & PEMM & PEN & PENO & PEP & PEQ & PESC & PET & PPE \\
\hline PEB & 0,915 & 0,756 & 0,794 & 0,391 & 0,598 & 0,590 & 0,427 & 0,394 & 1,000 & 0,741 & 1,000 & 0,542 \\
PEC & 0,915 & 0,756 & 0,794 & 0,391 & 0,598 & 0,590 & 0,427 & 0,394 & 1,000 & 0,741 & 1,000 & 0,542 \\
PEE & 0,745 & 0,594 & 0,837 & 0,349 & 0,512 & 0,230 & 0,167 & 0,294 & 1,000 & 0,644 & 1,000 & 0,474 \\
PEM & 0,694 & 0,520 & 0,657 & 0,490 & 0,485 & 0,450 & 0,440 & 0,999 & 0,717 & 1,000 & 1,000 & 0,758 \\
PEMM & 0,616 & 0,655 & 0,706 & 0,273 & 0,652 & 0,459 & 0,272 & 0,264 & 0,995 & 0,762 & 1,000 & 0,955 \\
PEN & 0,822 & 0,666 & 0,695 & 0,436 & 0,507 & 0,745 & 0,725 & 0,558 & 0,483 & 0,698 & 0,999 & 0,501 \\
PENO & 0,822 & 0,666 & 0,695 & 0,436 & 0,507 & 0,743 & 0,725 & 0,559 & 0,482 & 0,698 & 1,000 & 0,501 \\
PEP & 0,178 & 0,120 & 0,323 & 0,262 & 0,129 & 0,055 & 0,168 & 1,000 & 0,161 & 0,323 & 0,574 & 0,241 \\
PEQ & 0,700 & 0,586 & 0,619 & 0,252 & 0,474 & 0,330 & 0,134 & 0,186 & 1,000 & 0,549 & 0,708 & 0,406 \\
PESC & 0,679 & 0,528 & 0,65 & 0,474 & 0,502 & 0,480 & 0,441 & 0,954 & 0,722 & 1,000 & 1,000 & 0,804 \\
PET & 0,621 & 0,657 & 0,708 & 0,274 & 0,652 & 0,459 & 0,271 & 0,264 & 1,000 & 0,762 & 1,000 & 0,951 \\
PPE & 0,088 & 0,295 & 0,334 & 0,113 & 0,432 & 0,309 & 0,261 & 0,182 & 0,307 & 0,511 & 0,686 & 1,000 \\
\hline$e_{k}$ & 0,650 & 0,567 & 0,651 & 0,345 & 0,504 & 0,453 & 0,371 & 0,504 & 0,739 & 0,702 & 0,914 & 0,640 \\
\hline
\end{tabular}

Na Tabela 5 apresenta-se a ordenação segundo a eficiência cruzada. Verifica-se que o programa PET obteve o primeiro lugar, sendo que a eficiência cruzada foi muito parecida com a eficiência padrão obtida na primeira etapa do estudo. Isso conduz a uma melhor observação das diferenças entre a eficiência padrão e a eficiência cruzada de cada um dos programas. Nota-se que existem grandes diferenças entre essas duas eficiências, sendo a maior delas a do programa PEP, que pode ser explicada pelas avaliações dos diferentes programas na Tabela 4 (ver a coluna de PEP).

Pode-se ainda observar que o programa PEP foi avaliado com um índice baixo por quase todos os programas, sendo que a pior avaliação foi a do programa PPE $(0,182)$, o que significa que o programa PPE dá importância a variáveis que o programa PEP ignora. A mesma análise pode ser feita para todos os programas que tiveram baixa avaliação por parte de outros programas. O programa PET é o de melhor eficiência final, sendo que a avaliação por parte de outros programas é muito favorável e constante.

É importante notar que a matriz de avaliação cruzada (Tabela 4) pode ser muito útil para identificar parceiros ou programas com perfis semelhantes, no sentido em que a avaliação por parte desse programa seja uma eficiência consistente com a eficiência encontrada na primeira avaliação. Assim, o programa PEP pode identificar como parceiros, ou programas de perfil semelhante, os programa PESC e PEM. Por outro lado, podem-se igualmente identificar programas antagonistas. No caso de PEP são, entre outros, os programa PPE e PEQ (na verdade, quase todos os programas estão fornecendo baixa avaliação ao programa PEP). 
Tabela 5. Hierarquização dos programas de pós-graduação segundo a avaliação cruzada.

\begin{tabular}{cccc}
\hline Ordem & DMU & Eficiência cruzada & Eficiência DEA CCR \\
\hline $1^{\circ}$ & PET & 0,914 & 1,000 \\
$2^{\circ}$ & PEQ & 0,739 & 1,000 \\
$3^{\circ}$ & PESC & 0,702 & 1,000 \\
4 & PEE & 0,651 & 0,837 \\
$5^{\circ}$ & PEB & 0,650 & 0,915 \\
$6^{\circ}$ & PPE & 0,640 & 1,000 \\
$7^{\circ}$ & PEC & 0,567 & 0,756 \\
$8^{\circ}$ & PEMM & 0,504 & 0,652 \\
$9^{\circ}$ & PEP & 0,504 & 1,000 \\
$10^{\circ}$ & PEN & 0,453 & 0,745 \\
$11^{\circ}$ & PENO & 0,371 & 0,725 \\
$12^{\circ}$ & PEM & 0,345 & 0,490 \\
\hline
\end{tabular}

\section{Conclusões}

A Análise Envoltória de Dados mostrou-se uma ferramenta importante para a avaliação de programas de pósgraduação, ao serem consideradas múltiplas variáveis que apresentam relação causal, sem introduzir pesos arbitrários. Assim, os resultados obtidos independem de opiniões subjetivas, e quase sempre polêmicas, dos avaliadores, sejam internos ou externos.

Além disso, o uso da Avaliação Cruzada permitiu incrementar a discriminação da avaliação entre os programas, e, mais importante, considerar uma avaliação de conjunto na qual cada um programa avalia todos os outros, sem qualquer informação $a$ priori. Essa técnica adicional também permitiu identificar unidades parceiras e antagonistas, a partir da análise da matriz de eficiências cruzadas.

Os resultados deste artigo podem contribuir para o uso de uma nova estratégia para a avaliação no ensino, utilizando a Análise Envoltória de Dados (DEA) e a Avaliação Cruzada, em situações em que o consenso não seja possível. Não obstante, é aconselhável que a técnica da avaliação cruzada seja restrita a um conjunto fixo de unidades em análise, pois, por tratar-se de uma média, a retirada ou inclusão de uma unidade em análise pode afetar o resultado final da avaliação cruzada.

\section{Referências Bibliográficas}

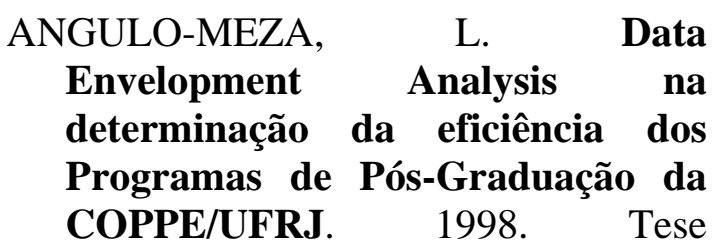
(Mestrado em Eng. de Produção) COPPE, Universidade Federal do Rio de Janeiro, Rio de Janeiro.

ANGULO-MEZA, L.; LINS, M.P.E. Review of Methods for Increasing Discrimination in Data Envelopment Analysis. Annals of Operations Research, v. 116, p. 225-242, 2002.

BOCLIN, R. Indicadores de Desempenho: Novas Estratégias da Educação Superior. Ensaio Avaliação e Políticas Públicas em Educação, n. 7, p. 299-308, 1999.

BANKER, R.D.; CHARNES, A.; COOPER, W.W. Some models for estimating technical scale inefficiencies in Data Envelopment Analysis. Management Science, v. 30, p. 1078-1092, 1984.

CHARNES, A.; COOPER, W.W.; RHODES, E. Measuring the efficiency of decision-making units. European Journal of Operational Research, v. 2, p. 429-444, 1978.

COOPER, W.W.; SEIFORD, L.M.; TONE, K. Data Envelopment Analysis: A Comprehensive Text with Models, Applications, References and DEA-Solver Software. Boston: Kluwer Academic Publishers, 2000. 
DOYLE, J. R.; GREEN, R. H. Efficiency and Cross-efficiency in DEA: Derivations, Meanings and Uses. Journal of the Operational Research Society, v. 45 n. 5, p. 567578, 1994.

GONZALEZ-ARAYA, M.C. Projeções não radiais em regiões fortemente eficientes da fronteira DEA Algoritmos e Aplicações, 2003. Tese (Doutorado em Engenharia de Produção) - COPPE, Universidade Federal do Rio de Janeiro, Rio de Janeiro.

GOMES, E.G.; SOARES DE MELLO, J.C.C.B.; SERAPIÃO, B.P.; LINS, M.P.E.; BIONDI, L.N. Avaliação de Eficiência de Companhias Aéreas Brasileiras: Uma Abordagem por Análise de Envoltória de Dados. In: SETTI, J.R.A.; LIMA JÚNIOR, O.F. (Eds.). Panorama Nacional da Pesquisa em Transportes 2001. Campinas: SP, v. 2, p. 125-133, 2001. GOMES, E.G.; SOARES DE MELLO, J.C.C.B.; $\quad$ ANGULO-MEZA,L.; LINS, M.P.E. Qualidade e Produtividade de Programas de PósGraduação em Engenharia. Anais do VIII Encontro de Educação em Engenharia, 2002.

LINS, M.P.E.; ANGULO-MEZA, L. Análise Envoltória de Dados e perspectivas de integração no ambiente de Apoio à Decisão. Rio de Janeiro: Editora da COPPE, 2000.

SOARES DE MELLO, J.C.C.B.; GOMES, E.G.; SOARES DE MELLO, M.H.C.; LINS, M.P.E., Seleção de Variáveis para utilização de Análise Envoltória de Dados como ferramenta Multicritério: uma aplicação em Educação. Anais do XXI ENEGEP - Encontro Nacional de Engenharia de Produção e VII International Conference on Industrial Engineering and Operations Management, Salvador, 2001a.

SOARES DE MELLO, J.C.C.B.; LETA, F.R.; FERNANDES, A.J.S.; VAZ, M.R.; SOARES DE MELLO, M.H.C.; BARBEJAT, M.E.R.P. Avaliação Qualitativa e Quantitativa: uma Metodologia de Integração. Ensaio - Avaliação e Políticas Públicas em Educação, v. 9, n. 31, p. 237-251, 2001b.

SOARES DE MELO, J.C.C.B., GOMES, E.G., ANGULO-MEZA, L., SOARES DE MELLO, M.H.C., Análise Envoltória de Dados na Avaliação da Produtividade Científica de Programas de Pós-Graduação em Engenharia. Anais do XXII Encontro Nacional de Engenharia de Produção, 2002a.

SOARES DE MELO, J.C.C.B., LINS, M.P.E., SOARES DE MELLO, M.H.C., GOMES, E.G., Evaluating the Performance of Calculus Classes Using Operational Research Tools. European Journal of Engineering Education, v. 27, n. 2, 2002b. 